\title{
ERRATUM
}

\section{Erratum to: Primary Nasal Tip Surgery: A Conservative Approach}

\author{
Marco Klinger · Fabio Caviggioli $\cdot$ Davide Forcellini • \\ Valeria Bandi · Luca Maione • Valeriano Vinci • Angelo Virgilio Pagliari • \\ Francesco Klinger $\cdot$ Riccardo Francesco Mazzola
}

Published online: 26 January 2013

(c) Springer Science+Business Media New York and International Society of Aesthetic Plastic Surgery 2013

Erratum to: Aesth Plast Surg (2012) 36:485-490
DOI 10.1007/s00266-011-9839-0

"Fondazione IRCCS Ca' Granda Ospedale Maggiore

Policlinico" was added to the last author's affiliation.

The online version of the original article can be found under doi:10.1007/s00266-011-9839-0.

M. Klinger · D. Forcellini · V. Bandi · L. Maione · V. Vinci

U.O. Chirurgia Plastica 2, Dipartimento di Medicina

Traslazionale, Università degli Studi di Milano, IRCCS Istituto

Clinico Humanitas, Via A. Manzoni 56, 20089 Rozzano, Milano,

Italy

e-mail: marco.klinger@humanitas.it

F. Caviggioli $\cdot$ F. Klinger

Cattedra di Chirurgia Plastica, MultiMedica Holding S.p.A.,

U.O.C. Chirurgia Plastica, Università degli Studi di Milano,

Sesto San Giovanni, Milan, Italy

A. V. Pagliari

Unità Operativa di Otorinolaringoiatria, Ospedale di Crema,

Crema, Italy

R. F. Mazzola $(\square)$

Dipartimento di Scienze Chirurgiche Specialistiche, Fondazione IRCCS Ca' Granda Ospedale Maggiore Policlinico, Università degli Studi di Milano, Milan, Italy

e-mail: riccardo.mazzola@fastwebnet.it 